Georgian Mathematical Journal

Volume 11 (2004), Number 1, 93-98

\title{
VARIETIES OF UNIVERSAL ALGEBRAS WITH NORMAL LOCAL PROJECTIONS
}

\author{
Z. JANELIDZE
}

\begin{abstract}
In this paper we characterize varieties of universal algebras in which every fibre $\operatorname{Pt}(B)$ of the fibration of points has normal product projections, i.e., $(X \times Y) / X \approx Y$ holds in every fibre $\operatorname{Pt}(B)$.
\end{abstract}

2000 Mathematics Subject Classification: 18A20, 18A30, 18D30, 08B05, $08 \mathrm{~B} 25$.

Key words and phrases: Fibration of points, product projection, normal epimorphism, Mal'tsev category, Mal'tsev variety, Jónsson-Tarski variety, subtractive variety.

\section{INTRODUCTION}

As defined in [5], a pointed category $\mathbf{C}$ is said to have normal projections if every product projection in $\mathbf{C}$ is a normal epimorphism; equivalently, for any two objects $X$ and $Y$ in $\mathbf{C}$, the canonical morphism $(X \times Y) / X \rightarrow Y$ is an isomorphism. In [5] we have characterized the varieties of universal algebras satisfying this condition. In particular, it holds in every Jónsson-Tarski variety [7] (more generally, in every unital category in the sense of D. Bourn [2]) and in every pointed subtractive variety in the sense of A. Ursini [11].

In the present paper, in a similar way as in [5], we will characterize the varieties satisfying the following 'local version' of the above condition.

Definition 0.1. A category $\mathbf{C}$ is said to have normal local projections if every fibre $\operatorname{Pt}(B)$ of the fibration of points has normal projections.

If $\mathbf{C}$ is a pointed category, then this condition implies the previous one. This is because in the case $B=0$ the fibre $\operatorname{Pt}(B)=\operatorname{Pt}(0)$ is isomorphic to $\mathbf{C}$. The converse is not true, as, for instance, the category of monoids (commutative or not) does not satisfy the condition of Definition 0.1. However, this condition holds in every Mal'cev category [4], and hence in every Mal'cev variety [10]. As we will show using our characterization theorem, it also holds in the category of lattices and in the category of implication algebras.

\section{The Characterization Theorem}

Let $\mathbf{C}$ be a category with finite limits and coequalizers. Let $B$ be an object in $\mathbf{C}$; recall the following:

(a) The objects in $\operatorname{Pt}(B)$ are all triples $(A, \alpha, \beta)$, where $A$ is an object in $\mathbf{C}$ and $\alpha: A \rightarrow B, \beta: B \rightarrow A$ morphisms with $\alpha \beta=1_{B}$. A morphism $f:(A, \alpha, \beta) \rightarrow$ 
$\left(A^{\prime}, \alpha^{\prime}, \beta^{\prime}\right)$ in $\operatorname{Pt}(B)$ is a morphism $f: A \rightarrow A^{\prime}$ in $\mathbf{C}$ such that $\alpha^{\prime} f=\alpha$ and $f \beta=\beta^{\prime}$.

(b) $\operatorname{Pt}(B)$ is a pointed category, with the zero object $\left(B, 1_{B}, 1_{B}\right)$. The zero morphism $0:(A, \alpha, \beta) \rightarrow\left(A^{\prime}, \alpha^{\prime}, \beta^{\prime}\right)$ (i.e. the morphism that factors through the zero object) is the composite $\beta^{\prime} \alpha$.

(c) Consider the pullback

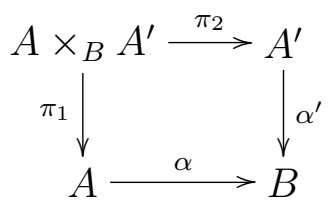

and let $\left[\beta, \beta^{\prime}\right]: B \rightarrow A \times_{B} A^{\prime}$ be the canonical morphism. The triple $\left(A \times_{B}\right.$ $\left.A^{\prime}, \alpha \pi_{1},\left[\beta, \beta^{\prime}\right]\right)$ is the product of $(A, \alpha, \beta)$ and $\left(A^{\prime}, \alpha^{\prime}, \beta^{\prime}\right)$ in $\operatorname{Pt}(\mathrm{B})$, with the product projections $\pi_{1}$ and $\pi_{2}$.

By Definition 0.1, $\mathbf{C}$ has normal local projections if and only if for any object $B$ in $\mathbf{C}$ and $X=(A, \alpha, \beta), Y=\left(A^{\prime}, \alpha^{\prime}, \beta^{\prime}\right)$ in $\operatorname{Pt}(B)$, the product projection $\pi_{2}: X \times Y \rightarrow Y$ is a normal epimorphism, i.e. $\pi_{2}$ is the cokernel of the product injection $\iota_{1}=\left[1_{X}, 0\right]: X \rightarrow X \times Y$. Since the forgetful functor $\operatorname{Pt}(B) \rightarrow \mathbf{C}$ creates coequalizers, this means that

$$
A \stackrel{\left[1_{A}, \beta^{\prime} \alpha\right]}{\underset{\left[\beta, \beta^{\prime}\right] \alpha}{\longrightarrow}} A \times \times_{B} A^{\prime} \stackrel{\pi_{2}}{\longrightarrow} A^{\prime}
$$

is a coequalizer diagram in $\mathbf{C}$; if $\mathbf{C}$ is a variety of universal algebras, then this is equivalent to the following:

$$
\forall_{a \in A, a^{\prime} \in A^{\prime}, \alpha(a)=\alpha^{\prime}\left(a^{\prime}\right)} \exists_{n \in \mathbf{N}}\left(\left(a, a^{\prime}\right),\left(\beta \alpha(a), a^{\prime}\right)\right) \in R^{n},
$$

where $\mathbf{N}$ is the set of natural numbers, and $R$ is the smallest reflexive and symmetric homomorphic relation on $A \times_{B} A^{\prime}$ containing the relation $\left\{\left(\left(a, \beta^{\prime} \alpha(a)\right)\right.\right.$, $\left.\left.\left(\beta \alpha(a), \beta^{\prime} \alpha(a)\right)\right) \mid a \in A\right\}$.

Let $F[x, y]$ and $F[x]$ be the free algebras on the sets $\{x, y\}$ and $\{x\}$, respectively, and let $p: F[x, y] \rightarrow F[x]$ be the morphism induced by the unique function $\{x, y\} \rightarrow\{x\}$. We write $F[x, y] \times_{F[x]} F[x, y]$ to denote the pullback

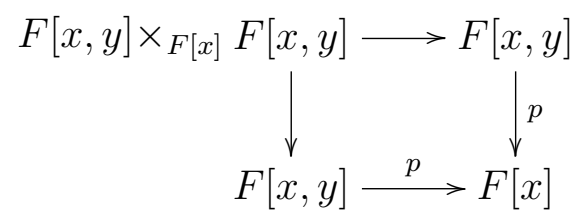

Proposition 1.1. The following conditions are equivalent:

1. C has normal local projections;

2. There exists a natural number $n$ such that $((y, y),(x, y)) \in Q^{n}$, where $Q$ is the reflexive homomorphic relation on $F[x, y] \times_{F[x]} F[x, y]$ generated by the set $\{((y, x),(x, x)),((x, x),(y, x))\}$. 
Proof. It is easy to see that condition 2 of Proposition 1.1 is a special case of (1), where $A=A^{\prime}=F[x, y], B=F[x], \alpha=\alpha^{\prime}=p$ and $\beta=\beta^{\prime}=i: F[x] \rightarrow F[x, y]$ is the inclusion. This leaves to prove that condition 2 implies condition 1. Suppose 2 holds; we have to show that (1) holds (for all $A, A^{\prime}, \ldots$ ). Let $a \in A$ and $a^{\prime} \in A^{\prime}$, with $\alpha(a)=\alpha^{\prime}\left(a^{\prime}\right)$, and let $R$ be as in (1); we must show that $\left(\left(a, a^{\prime}\right),\left(\beta \alpha(a), a^{\prime}\right)\right)$ is in $R^{n}$. Let $f: F[x, y] \rightarrow A$ and $g: F[x, y] \rightarrow A^{\prime}$ be the morphisms of algebras induced by the assignments $y \mapsto a, x \mapsto \beta \alpha(a)$ and $y \mapsto a^{\prime}, x \mapsto \beta^{\prime} \alpha^{\prime}\left(a^{\prime}\right)=\beta^{\prime} \alpha(a)$, respectively, and let $h: F[x] \rightarrow B$ be the morphism which takes $x$ to $\alpha(a)=\alpha^{\prime}\left(a^{\prime}\right)$. Then there is an induced morphism

$$
F[x, y] \times_{F[x]} F[x, y] \stackrel{c}{\longrightarrow} A \times_{B} A^{\prime}
$$

It is easy to see that the image of $Q^{n}$ under $c \times c$ lies in $R^{n}$; hence, from $((y, y),(x, y)) \in Q^{n}$ we obtain $\left(\left(a, a^{\prime}\right),\left(\beta \alpha(a), a^{\prime}\right)\right)=(c(y, y), c(x, y)) \in R^{n}$.

Theorem 1.2. A variety $\mathbf{C}$ has normal local projections if and only if the corresponding theory contains

- binary terms $t_{1}, \ldots, t_{m}, u_{1}, \ldots, u_{m}$;

- $(m+2)$-ary terms $v_{1}, \ldots, v_{n}$;

and the following identities hold in $\mathbf{C}$ :

- $y=v_{1}\left(t_{1}(x, y), \ldots, t_{m}(x, y), y, x\right)=v_{n}\left(t_{1}(y, x), \ldots, t_{m}(y, x), y, x\right)$;

- $y=v_{i}\left(u_{1}(x, y), \ldots, u_{m}(x, y), x, x\right)$, for each $i \in\{1, \ldots, n\}$.

- $v_{i+1}\left(t_{1}(x, y), \ldots, t_{m}(x, y), y, x\right)=v_{i}\left(t_{1}(x, y), \ldots, t_{m}(x, y), x, y\right)$, for each $i \in\{1, \ldots, n-1\}$;

- $t_{j}(x, x)=u_{j}(x, x)$, for each $j \in\{1, \ldots, m\}$.

Proof. According to Proposition 1.1, C has normal local projections if and only if the pair $((y, y),(x, y))$ is in $Q^{n}$. This is the case if and only if there exist $s_{1}, \ldots, s_{n+1} \in F[x, y]$ such that $s_{1}=y, s_{n+1}=x$, and for each $i \in\{1, \ldots, n\}$ the pair $\left(\left(s_{i}, y\right),\left(s_{i+1}, y\right)\right)$ is in $Q$. The latter holds if and only if

$$
\begin{gathered}
\left(s_{i}, y\right)=v_{i}\left(\left(t_{1}, u_{1}\right), \ldots,\left(t_{m}, u_{m}\right),(y, x),(x, x)\right), \\
\left(s_{i+1}, y\right)=v_{i}\left(\left(t_{1}, u_{1}\right), \ldots,\left(t_{m}, u_{m}\right),(x, x),(y, x)\right),
\end{gathered}
$$

for binary terms $t_{1}, \ldots, t_{m}, u_{1}, \ldots, u_{m}$ and some $(m+2)$-ary term $v_{i}$, such that $t_{j}(x, x)=u_{j}(x, x)$ for each $j \in\{1, \ldots, m\}$. Equivalently, $\left(\left(s_{i}, y\right),\left(s_{i+1}, y\right)\right) \in Q$ if and only if

$$
s_{i}=v_{i}\left(t_{1}, \ldots, t_{m}, y, x\right), \quad s_{i+1}=v_{i}\left(t_{1}, \ldots, t_{m}, x, y\right), \quad y=v_{i}\left(u_{1}, \ldots, u_{m}, x, x\right) .
$$

Moreover, we can assume that $t$ 's, $u$ 's and the number $m$ are the same for each $i \in\{1, \ldots, n\}$. Reformulating this without $s$ 's, which are expressed by $v$ 's, we obtain the desired characterization.

Remark 1.3. condition 2 of Proposition 1.1 used to prove this theorem is similar to 2(c) of [5] used to prove Theorem 3 in [5], which characterizes pointed varieties with normal projections. Moreover, if we write 0's in the place of $x$ 's in Theorem 1.2, we obtain a reformulation of Theorem 3 in [5]. 
Remark 1.4. As it has already been mentioned, the category of monoids, commutative or not, does not have normal local projections. This is easy to see from the identities in the characterization theorem.

Example 1.5. Let $\mathbf{C}$ be a variety of universal algebras for which we could take $n=1$ in 1.2 . The theory corresponding to such $\mathbf{C}$ has an $(m+2)$-ary term $v$ and binary terms $t_{1}, \ldots, t_{m}, u_{1}, \ldots, u_{m}$, which satisfy the identities

$$
\begin{aligned}
& y=v\left(t_{1}(x, y), \ldots, t_{m}(x, y), y, x\right)=v\left(t_{1}(y, x), \ldots, t_{m}(y, x), y, x\right), \\
& y=v\left(u_{1}(x, y), \ldots, u_{m}(x, y), x, x\right), \\
& t_{j}(x, x)=u_{j}(x, x) \text { for each } j \in\{1, \ldots, m\} .
\end{aligned}
$$

If, in addition, for each $j$, every one of the terms $t_{j}=t_{j}\left(x_{1}, x_{2}\right)$ and $u_{j}=$ $u_{j}\left(x_{1}, x_{2}\right)$ is equal to either the first variable $x_{1}$, or the second variable $x_{2}$, an easy argument shows that we can rewrite these identities as

$$
y=w(x, x, y, y, y, x)=w(y, y, x, x, y, x)=w(x, y, y, x, x, x) .
$$

If the term $w=w\left(x_{1}, x_{2}, x_{3}, x_{4}, x_{5}, x_{6}\right)$ depends only on $x_{3}, x_{4}$ and $x_{5}$, then we can write $w\left(x_{1}, x_{2}, x_{3}, x_{4}, x_{5}, x_{6}\right)=p\left(x_{3}, x_{4}, x_{5}\right)$ and the identities become

$$
y=p(x, x, y)=p(y, x, x),
$$

which defines a Mal'cev variety [10]. On the other hand, $w\left(x_{1}, x_{2}, x_{3}, x_{4}, x_{5}, x_{6}\right)=$ $p\left(x_{2}, x_{3}, x_{5}\right)$ would give

$$
y=p(x, y, y)=p(y, x, y)=p(y, y, x) .
$$

An example of a variety which contains such $p$ (called the Jónsson term, see [6], [8]) is the category of lattices, where we could take $p(x, y, z)=(x \vee y) \wedge(y \vee$ $z) \wedge(z \vee x)$

Example 1.6. An implication algebra is a triple $(A, 1, \rightarrow)$, where $A$ is a set, 1 is a 0 -ary operation on $A$, and $\rightarrow$ is a binary operation on $A$ satisfying:

$$
\begin{aligned}
& (y \rightarrow x) \rightarrow y=y, \\
& (y \rightarrow x) \rightarrow x=(x \rightarrow y) \rightarrow y, \\
& y \rightarrow(x \rightarrow z)=x \rightarrow(y \rightarrow z), \\
& x \rightarrow x=1
\end{aligned}
$$

(see [1],[9]). The variety of implication algebras has normal local projections. Indeed, take $n=m=2, t_{1}(x, y)=u_{1}(x, y)=u_{2}(x, y)=y, t_{2}(x, y)=x$ in 1.2 , then the identities there become

$$
\begin{aligned}
& y=v_{1}(y, x, y, x)=v_{2}(x, y, y, x), \\
& y=v_{1}(y, y, x, x)=v_{2}(y, y, x, x), \\
& v_{2}(y, x, y, x)=v_{1}(y, x, x, y) .
\end{aligned}
$$

Rewriting these identities for $v_{1}(x, y, z, t)=v_{2}(y, x, z, t)=p(x, z, t)$, we get

$$
\begin{aligned}
& y=p(y, y, x)=p(y, x, x), \\
& p(x, y, x)=p(y, x, y) .
\end{aligned}
$$

Thus, if a variety $\mathbf{C}$ contains a ternary term $p$ satisfying the above identities, then $\mathbf{C}$ has normal local projections. In the case of the variety of implication algebras, we can put $p(x, y, z)=(y \rightarrow z) \rightarrow x$. 


\section{Additional Remarks}

Let $\mathbf{C}$ be a pointed category with finite limits, containing all intersections of subobjects.

Definition 2.1. Let $n$ be a natural number. A regular epimorphism $f: A \rightarrow$ $B$ in $\mathbf{C}$ is said to be $n$-normal if the kernel relation of $f$ is $S^{n}$, where $S$ is the symmetric and reflexive relation on $A$, generated by the relation $(\operatorname{ker}(f), 0)$ : $\operatorname{Ker}(f) \rightrightarrows A$.

For $n=1$ we can formulate this definition in a pointed category $\mathbf{C}$ having just finite limits as follows:

Definition 2.2. A regular epimorphism $f: A \rightarrow B$ in $\mathbf{C}$ is said to be 1-normal, if the canonical morphisms

$$
\begin{gathered}
\operatorname{Ker}(f) \stackrel{(\operatorname{ker}(f), 0)}{\longrightarrow} A \times_{B} A, \quad \operatorname{Ker}(f) \stackrel{(0, \operatorname{ker}(f))}{\longrightarrow} A \times_{B} A, \\
A \stackrel{\left(1_{A}, 1_{A}\right)}{\longrightarrow} A \times_{B} A
\end{gathered}
$$

are strongly epimorphic.

Using Proposition 1.1 above and Proposition 2 of [5], we obtain

Corollary 2.3. A variety $\mathbf{C}$ has normal local projections if and only if there exists a natural number $n$ such that every fibre $\operatorname{Pt}(B)$ of the fibration of points has n-normal product projections. A pointed variety $\mathbf{C}$ has normal projections if and only if for some $n, \mathbf{C}$ has n-normal product projections.

In particular, pointed varieties with 1-normal product projections are exactly Jónsson-Tarski varieties [7]. This is a special case of the proposition below, which follows easily from Theorem 1.2.13 and Proposition 1.2.17 of [3].

Proposition 2.4. A pointed category $\mathbf{C}$ with finite limits is unital if and only if it has 1-normal product projections.

Since Mal'cev categories are exactly those categories for which every fibre $\operatorname{Pt}(B)$ of the fibration of points is a unital category (see [2]), we obtain:

Corollary 2.5. A category $\mathbf{C}$ with finite limits is a Mal'cev category if and only if every fibre $\operatorname{Pt}(B)$ of the fibration of points has 1-normal product projections.

Let us assign to each variety $\mathbf{C}$ with normal local projections the least natural number $n$ such that all fibres of the fibration of points have $n$-normal product projections. Then by 2.5, Mal'cev varieties are exactly those varieties for which $n=1$. From this it also follows that in other situations considered in 1.5 , and in particular for the category of lattices, the corresponding $n$ is equal to 2 . In the case of the varieties considered in 1.6 , one has $2 \leq n \leq 4$. 


\section{REFERENCES}

1. J. C. Аввот, Implication algebras. Bull. Math. Soc. Sci. Math. R. S. Roumanie 11(59)(1967), 3-23 (1968).

2. D. Bourn, Mal'cev categories and fibration of pointed objects. The European Colloquium of Category Theory (Tours, 1994). Appl. Categ. Structures 4(1996), No. 2-3, 307-327.

3. D. Bourn and F. Borceux, A conceptual entry to general algebra. To appear.

4. A. Carboni, J. Lambek, and M. C. Pedicchio, Diagram chasing in Mal'cev categories. J. Pure Appl. Algebra 69(1990), 271-284.

5. Z. JANELIDZE, Characterization of pointed varieties of universal algebras with normal projections. Theory Appl. Categ. 11(2003), No. 9, 212-214.

6. B. Jónsson, Algebras whose congruence lattices are distributive. Math. Scand. 21(1967), $110-121$.

7. B. Jónsson and A. TARSKI, Direct decompositions of finite algebraic systems. Notre Dame Mathematical Lectures, no. 5. University of Notre Dame, Notre Dame, Ind., 1947.

8. K. KAARli and R. MCKenzie, Affine complete varieties are congruence distributive. Algebra Universalis 38(1997), 329-354.

9. J. G. Pitкethly, Endoprimal implication algebras. Algebra Universalis 41(1999), 201211.

10. J. D. H. Smith, Mal'cev varieties. Lecture Notes in Mathematics 554, Springer-Verlag, Berlin-Heidelberg, 1976.

11. A. Ursini, On subtractive varieties, I. Algebra Universalis 31(1994), 204-222.

(Received 17.04.2003)

Author's address:

Faculty of Mechanics and Mathematics

I. Javakhishvili Tbilisi State University

2, University St., Tbilisi 0143

Georgia 